\title{
Integrating high-speed videos in capture-mark-recapture studies of insects
}

\author{
Rassim Khelifa ${ }^{1}$, Hayat Mahdjoub ${ }^{1}$, Leithen M'Gonigle ${ }^{2}$, and Claire Kremen ${ }^{1}$ \\ ${ }^{1}$ The University of British Columbia \\ ${ }^{2}$ Simon Fraser University
}

November 24, 2020

\begin{abstract}
Capture-mark-recapture (CMR) studies have been used extensively in ecology and evolution. While it is feasible to apply CMR in some animals, it is considerably more challenging in small fast-moving species such as insects. In these groups, low recapture rates can bias estimates of demographic parameters, thereby, handicapping effective management of wild populations. Here we use high-speed videos (HSV) of the adults of two large dragonfly species that rarely land and, thus, are particularly challenging for CMR studies. We specifically test whether HSV, compared to conventional eye observations, increases the "resighting" rates and improves the certainty of the estimates of survival rate, and the effects of demographic covariates on survival rates. We show that the use of HSV increases the number of resights substantially. HSV improved our estimates of resighting and survival probability which were either under- or overestimated with the conventional observations. HSV increased the accuracy of the estimates of effect sizes of important covariates (age and body size). Integrating HSV in CMR of highly mobile animals is valuable because it is easy, non-invasive, and has the potential to improve demographic estimates. Hence, it opens the door for a wide range of research possibilities on species that are traditionally difficult to monitor, including within insects, birds, and mammals.
\end{abstract}

\section{Introduction}

Capture-mark-recapture (CMR) is a widely used technique to address fundamental questions in animal ecology, evolution, and biological conservation (Pradel, 1996; Lettink \& Armstrong, 2003; Amstrup, McDonald, \& Manly, 2010). It permits making powerful inferences about population size, survival rate, recruitment, dispersal, as well as predicting the effects of intrinsic (e.g. body size, age, disease) and extrinsic factors (e.g. temperature, precipitation, food) (Conn \& Cooch, 2009; Martins et al., 2011; Hassall, Sherratt, Watts, \& Thompson, 2015; Rose, Wylie, Casazza, \& Halstead, 2018) on those parameters. While marking or tagging animals can be relatively easy, recapturing marked individuals is often a challenging process, even in systems where individuals do not disperse. These difficulties are enhanced for highly mobile animals such as mammals, birds, and insects (Dickinson, Lehmann, \& Sane, 1999; Muijres, Johansson, Bowlin, Winter, \& Hedenström, 2012).

Insect species can be good model organisms for CMR studies (Hagler \& Jackson, 2001). Besides their smaller size which allows convenient capture, handling, and marking, they also have a short life cycle, conspicuous behavior, small home range size, and fixed adult size. Identifying a marked individual often requires immobilizing it, as small size and rapid speed means that marks are typically difficult to detect for an insect that is in motion. While repeated physical captures of individuals are one method that have often been used to accomplish identification of marked individuals (Hagler \& Jackson, 2001), repeat captures of insects may alter their behavior and/or cause injuries, thereby affecting their survival (Morton, 1982; Cordero, Perez, \& Andres, 2002). Telemetry is another possible solution, but it is still technologically challenging and costly 
to miniaturize transmitters to a size that is suitable for most insects (Daniel Kissling, Pattemore, \& Hagen, 2014). Consequently, most CMR studies have been carried out with conventional direct observations of marks on insects, but theses often yield low recapture rates and, subsequently, produce unreliable demographic and/or dispersal estimates (Service, 1993). Such poor estimates can hinder conservation planning (Hammill \& Clements, 2020).

One of the most frequent insect groups in CMR studies is odonates (dragonflies and damselflies) (CorderoRivera \& Stoks, 2008). Usually, an alphanumeric code is written on the wing with a permanent marker. After marking, some species of odonates (belonging to Aeshnidae) are particularly challenging to resight, because they rarely perch, instead spending most of their time in flight, where they are too fast for the human eye (Corbet, 1999). The use of conventional visual observations leads to low resighting rates. To overcome this impediment, a tool that ideally does not require the physical capture of individuals to identify dragonflies in flight is needed. One such potential tool is high-speed video (HSV) cameras, which capture videos with a high enough frame rate that one can slow down the motion of the individual and wings (Li, Zheng, Pan, \& $\mathrm{Su}, 2018$ ), thereby allowing for identification of the mark. HSV has thus the potential to gather data that cannot be obtained with conventional visual observations.

HSV have been used in the study of animal behavior, particularly during the last few decades. Whether it is flying, running, swimming, feeding or drinking, high-speed videos have enabled scientists to examine diverse behaviors of various taxa meticulously (Altshuler, Dudley, \& McGuire, 2004; Maie, Wilson, Schoenfuss, \& Blob, 2009; Reis, Jung, Aristoff, \& Stocker, 2010; Marras et al., 2015). The rapid recent uptake of this technology in ecology is due to the substantial decrease in the cost of such cameras, the decline in the camera's size and weight making it suitable for field studies, and the improved video capabilities in duration and quality (Steen, 2014; Garbin, 2018; Lailvaux, 2018). Although HSVs have been used to study various aspects of animal behavior, thus far, HSV not been integrated into CMR studies of highly mobile animals.

Here we assess how HSV can contribute to the resighting of marked individuals and ask whether such a method can improve estimates of demographic parameters. Using populations at artificial ponds, we carried out CMR on two large North American dragonflies, Anax junius and Rhionaeschna multicolor(Paulson, 2009), and then used the Cormack-Jolly-Seber model to compare the resighting and survival rate of the capture history generated by the conventional visual method (CV: direct observation with the naked eye) to that generated by combining $\mathrm{CV}$ with high-speed video (CV+HSV). Further, we tested whether HSV improves our ability to estimate the effect sizes of covariates that might impact survival, such as body size and age.

\section{Material and methods}

Study site

The study was conducted in the experimental ponds of the University of British Columbia, Vancouver, Canada ( $\left.49^{\circ} 14^{\prime} 56^{\prime \prime} \mathrm{N}, 123^{\circ} 14^{\prime} 02^{\prime \prime} \mathrm{W}\right)$. The site has a total of 20 artificial ponds, each of dimensions $15 \times 25 \mathrm{~m}^{2}$, arrayed across an area of $130 \times 100 \mathrm{~m}^{2}$. The two dominant species we studied, Anax junius and Rhionaeschna multicolor, are among the largest and highly mobile dragonflies in North America (Paulson, 2009).

Field sampling

We carried out daily CMR between $6^{\text {th }}$ June and $24^{\text {th }}$ July, 2020 from 11:00 to 17:00 by capturing mature individuals with hand nets and marking them with a permanent marker on the hind wing. Two observers continuously walked across the study site to capture unmarked individuals and tag recovery. Before releasing individuals at the point of capture, hind wing length (surrogate of body size) was measured with an electronic caliper to the nearest $0.01 \mathrm{~mm}$. We used two resighting methods: (1) conventional observations with the naked eyes (CV), and (2) high-speed videos using the Sony RX10 Mark4 with 250 fps high-frame-rate mode, which is 8-10 times more frames than normal cameras (allowing to slow the motion 8-10 times). The videos were later visualized for identifying the individual code (Fig. 1). We asked how the use of a high-speed camera increases the number of resightings relative to conventional observations alone, and, further, whether 
inclusion of camera data can improve estimates of survival and accompanying covariates (age and body size).

Important sampling notes

It is important to point out that most CV observations were made when the species were perched (rare behavior in reproductive sites), in copulation or oviposition, thus detectable with the naked eye. In these cases, it was not necessary to use HSV as the individual was already recorded with CV. HSV was typically used when individuals were in flight and nearly impossible to identify with the naked eye. Thus, observations acquired with CV and HSV cover different behaviors and rarely overlap. If an individual was observed with both CV and HSV on the same day, the HSV observation was discarded since it was then data that could have been obtained with CV. Instead of comparing CV with HSV, we are interested here in assessing how HSV can supplement data that could not be observed with $\mathrm{CV}$ and how this potential increase in the resolution of data improves demographic estimates. To accomplish this goal, we compare CV with CV+HSV.

Cormack Jolly-Seber model (CJS)

CJS model is a powerful tool to estimate the survival rate of marked individuals using live encounter data (Lebreton, Burnham, Clobert, \& Anderson, 1992). The model accounts for imperfect detections, gives estimates of the survival and resighting probabilities, and tests how the latter are determined by intrinsic factors such as age and body size and extrinsic factors such as the weather (Kéry \& Schaub, 2011). In odonates, many studies have used CJS to estimate recapture and survival rates (Cordero-Rivera \& Stoks, 2008).

We implemented the CJS model using the individual state-space formulation as described by Gimenez et al. (2007). The parameters involved in the likelihood $\operatorname{are} \Phi_{l, \tau}$ which is the probability that the individual $i$ survives to time occasion $t+1$ given that it is alive at time occasion $t(\mathrm{t}=1,2, \ldots, \mathrm{T}-1)$, and $p_{i, t}$ which is the probability of recapturing (resighting) individual $i$ at time occasion $t(\mathrm{t}=1,2, \ldots, \mathrm{T})$. The initial occasion where individual $i$ is observed is denoted $e_{i}$. The general state-space formulation of the CJS model includes the observation equation (1) and the state equation (2) where t [?] $e_{i}$ and $p_{i, e_{i}}=1$.

$Y_{i, t} \mid X_{i, t} \sim \operatorname{Bernoulli}\left(X_{i, t} p_{i, t}\right)(1)$

$X_{i, t+1} \mid X_{i, t} \sim \operatorname{Bernoulli}\left(X_{i, t} \Phi_{i, t}\right)(2)$

This approach disentangles between the actual demographic process and its observation in the field. The model works such that when individual $i$ is alive at time $t$, it has probability $p_{i, t}$ of being recorded and probability 1- $p_{i, t}$ of not being recorded, which translates into $\mathrm{Y}_{i, t}$ following Bernoulli $\left(p_{i, t}\right)$ given $\mathrm{X}_{\mathrm{i}, \mathrm{t}}=$ 1. When individual $i$ is dead at time occasion $\mathrm{t}$, it cannot be recorded, which translates into $\mathrm{Y}_{i, t}$ following Bernoulli (0) given $\mathrm{X}_{\mathrm{i}, \mathrm{t}}=0$. The implementation of the model was carried out using MCMC technique (Brooks, Catchpole, \& Morgan, 2000; Gimenez et al., 2009).

\section{CMR Data}

For each species, we constructed two types of binary capture history as a series of 1s (detection) and 0s (non-detection) for each individual. The first capture history involved only conventional observations, collected with the naked eye (CV dataset) whereas the second capture history included the combination of the conventional observations and high-speed video (CV+HSV dataset). Both datasets involved 39 timeoccasions (days) and the same number of marked individuals: a total of 107 A. junius (74 males and 33 females) and $152 R$. multicolor (96 males and 56 females).

Statistical analysis

All statistical analyses were carried out using R 3.4.0 (R Development Core Team, 2020). We implemented the MCMC algorithm using JAGS software (Plummer, 2003) within R. Specifically, we used the following packages: rjags (Plummer, 2019), R2jags (Su \& Yajima, 2020), and runjags (Denwood, 2016). We specified non-informative prior distributions for all parameters. Specifically, we used flat normal distributions for priors $(\operatorname{dnorm}(0,0.01))$ for both survival and resighting probabilities, as well as the intercept and slopes of covariates (age and wing length). We tested for the effect of sex on resighting probability and effects of 
age and wing length on survival probability. We ran three independent chains, each for 10,000 iterations, omitting the first 1000 as burn-in and thinning every 100 iterations to reduce autocorrelation. Values are mean \pm SE.

\section{Results}

Number of resightings

With CV observations, we resighted 28 (26.1\%) marked A. juniusand 25 (16.4\%) marked $R$. multicolor at least once. With CV+HSV observations, we resighted 46 (43\%) A. junius and 37 (24.3\%)R. multicolor individuals, which correspond to proportional increases in recaptures of $64.2 \%$ and $48 \%$, respectively.

\section{Resighting probability}

The average resighting probability estimated with CJS model was higher with CV+HSV than with CV in both species. In CV and CV+HSV, respectively, resighting probability was $0.05 \pm 0.01$ and $0.19 \pm 0.02$ in $A$. junius and $0.05 \pm 0.01$ and $0.06 \pm 0.01$ in $R$. multicolor. The effect of sex on resighting probability was revealed with CV + HSV but not with CV in $A$. junius, showing higher resighting probabilities for males than females (Fig. 2a). This sexual difference was not observed in $R$. multicolor (Fig. 2b).

\section{Survival probability}

We estimated a slight difference in the average survival rate when using $\mathrm{CV}$ versus $\mathrm{CV}+\mathrm{HSV}$ in both species. In A. junius, using CV and CV+HSV, respectively, we estimated average survival probability to be $0.90 \pm 0.02$ and $0.87 \pm 0.01$, and in $R$. multicolor, we estimated average survival probability to be $0.84 \pm 0.02$ and $0.88 \pm 0.03$.

The two methods showed differences in the pattern of survival probability across age and wing length. Both methods showed a slight increase in survival probability with age in A. junius, although the mean was slightly overestimated using CV (Fig. 3a). However, different patterns were found in $R$. multicolor where CV showed a constant survival probability across age whereas CV+HSV showed a negative effect of age (Fig. 3b). In A. junius, CV showed a positive effect of wing length on survival probability whereas CV+HSV showed the opposite pattern (Fig. 4a). No significant effect of wing length on survival was detected for $R$. multicolor using both methods (Fig. 4b).

\section{Credible intervals}

In both species, $\mathrm{CV}+\mathrm{HSV}$ produced estimates with smaller credible intervals for survival probability (Fig. $3,4)$. The credible intervals were 1.9 and 1.3 times smaller for the average resighting probability, and 2 and 1.3 times smaller for the average survival probability in $A$. junius and $R$. multicolor, respectively. Similar reduction of the credible intervals was observed in the analysis of the effect of age (Fig. 3) and wing length (Fig. 4) on survival probability.

\section{Discussion}

Our study demonstrates that the use of a high-speed camera increases the number of resightings and improves certainty of estimates of demographic parameters. Our analyses showed that HSV provided additional data that increased the number of resightings in our CMR data, improved estimates of resighting and survival probabilities, and reduced the uncertainty around the effect sizes of important covariates such as age and body size. Such improvements can make a big difference in the monitoring of survival rates, recruitment, or population size of wild populations. These findings highlight the importance of integrating HSV in CMR studies as an easy noninvasive tool to track wild populations of various animals including those of conservation concern. Such integration could open doors for various applications on population dynamics surveys of insects, as well as for a wide range of fast and highly mobile animals.

Implications of improving resighting estimates 
Understanding the reason for the non-detection of species is crucial to make behavioral and ecological inferences. In our study, HSV increased the resighting rate substantially in A. junius but not $R$. multicolor . This means that in the former species the marked individuals were present at the site but the observers were not able to detect them with the naked eye (false negative), whereas in the latter species the low resighting rate under both CV and CV+HSV was likely due to the absence of the individuals at the site. This interspecific difference in resighting rate is probably due to the difference in adult behavior where $R$. multicolor might be more dispersive thanA. junius (Conrad, Willson, Harvey, Thomas, \& Sherratt, 1999). Furthermore, our analysis of $A$. junius capture history showed that HSV might reveal sexual differences in resighting rates that could not be detected by CV. This discrepancy may lead to erroneous inferences about species behaviour and the dismissal of sexual differences in species movement and habitat use (Stoks, 2001; Fujiwara, Anderson, Neubert, \& Caswell, 2006). Moreover, exhaustive estimates of resighting rates may provide more power to detect traits that drives reproductive success in particular and evolution in general. In many insects, single individuals rarely land in breeding sites, whereas breeding pairs perch to copulate and lay eggs. This behavioral difference between breeding and non-breeding individuals poses a challenge for researchers interested in characterizing the fitness landscape and identifying how trait values correlate with fitness. Also, since resighting rate is an important component in demographic models, enhancing the estimation of resighting rates may lead to a more accurate evaluation of population size (Tufto et al., 2012), and ultimately to more effective management of wild populations (Hammill \& Clements, 2020). Therefore, the improvement of our estimates of resighting rates by HSV might resolve many issues when addressing ecological, conservation, and evolutionary questions.

\section{Implications of improving survival estimates}

Data on survival probability are crucial for demographic modeling (Carey, 1993). Our results show that HSV improved the estimates of survival probability in both species. Such improvement can increase the reliability of our predictions of population trends (Carey, 2001). Many scientists are interested in the effect of age on survival to investigate evolutionary theories related to senescence (Bonduriansky \& Brassil, 2002; Sherratt et al., 2010; Sherratt, Hassall, Laird, Thompson, \& Cordero-Rivera, 2011; Zajitschek, Zajitschek, \& Bonduriansky, 2020). While this is theoretically feasible with CMR of wild population of insects, field studies based on the naked eye yield low-resolution data (low resighting rates) which prevents accurate predictions. In our study, we showed that HSV reduced the uncertainty around survival estimates across age and made the age-dependent survival pattern more detectable. Similarly, HSV improved our estimates of body size effects on survival probability. This is particularly important because body size has been the focus of many studies in evolutionary biology and ecology (LaBarbera, 1989; Blanckenhorn, 2000; Ozgul, Bateman, English, Coulson, \& Clutton-Brock, 2014).

\section{Conclusion}

We believe that HSV is a valuable noninvasive, economic, and practical tool in CMR. The advantages of using HSV include improving data quality, minimizing the physical damage to the study species, reducing sampling efforts, and saving money that would be allocated to hiring field assistants. The integration of HSV in CMR is timely because there have been remarkable technological advances in high-speed video capabilities during the past decade where cameras can generate high-quality HSV, capture longer videos, zoom to far distances, process the recording rapidly, and store large files. Nowadays, DSLR cameras have high frame-rate video modes at reasonably low costs (Steen, 2014). HSV could also be used in CMR of highly-mobile birds and mammals. Future methodological integration of high-speed camera might be in camera-trapping-based studies (Rico-Guevara \& Mickley, 2017) to investigate demographic parameters, dispersal, home range, and behavior.

\section{Acknowledgements}

We are grateful to Dolph Schluter for giving access to the UBC experimental ponds. We also thank Gaurav Singh-Varma for assistance in the field. This work is funded by the Swiss National Science Foundation (P400PB_191139) and the Work-Learn International Undergraduate Assistance program. 


\section{Authors' contributions}

RK conceived the idea; RK and HM did the field work; RK and LM analyzed the data; RK led the writing of the paper with contribution from all authors who gave final approval for publication.

\section{Data availability}

The full dataset and R code will be deposited in Dryad repository when the paper is accepted.

\section{Competing interests}

Authors have no competing interests, financial or otherwise, to declare.

\section{References}

Altshuler, D. L., Dudley, R., \& McGuire, J. A. (2004). Resolution of a paradox: hummingbird flight at high elevation does not come without a cost. Proceedings of the National Academy of Sciences, 101 (51), 17731-17736. doi:doi: 10.1073/pnas.0405260101

Amstrup, S. C., McDonald, T. L., \& Manly, B. F. (2010). Handbook of capture-recapture analysis : Princeton University Press.

Blanckenhorn, W. U. (2000). The evolution of body size: what keeps organisms small? The Quarterly Review of Biology, 75 (4), 385-407. doi:doi: 10.1086/393620

Bonduriansky, R., \& Brassil, C. E. (2002). Rapid and costly ageing in wild male flies. Nature, 420 (6914), 377-377. doi:10.1038/420377a

Brooks, S. P., Catchpole, E. A., \& Morgan, B. J. (2000). Bayesian animal survival estimation.Statistical Science, 15 (4), 357-376.

Carey, J. R. (1993). Applied demography for biologists: with special emphasis on insects : Oxford University Press.

Carey, J. R. (2001). Insect biodemography. Annual Review of Entomology, 46 (1), 79-110. doi:doi: 10.1146/annurev.ento.46.1.79

Conn, P. B., \& Cooch, E. G. (2009). Multistate capture-recapture analysis under imperfect state observation: an application to disease models. Journal of Applied Ecology, 46 (2), 486-492. doi:doi: 10.1111/j.13652664.2008.01597.x

Conrad, K., Willson, K., Harvey, I., Thomas, C., \& Sherratt, T. (1999). Dispersal characteristics of seven odonate species in an agricultural landscape. Ecography, 22 (5), 524-531. doi:doi: 10.1111/j.16000587.1999.tb01282.x

Corbet, P. S. (1999).Dragonflies: behaviour and ecology of Odonata . Colchester: Harley Books.

Cordero-Rivera, A., \& Stoks, R. (2008). Mark-recapture studies and demography. In A. Cordoba-Aguilar (Ed.), Dragonflies and damselfies: Model organisms for ecological and evolutionary research (pp. 7-20). Oxford: Oxford University Press.

Cordero, A., Perez, F. E., \& Andres, J. (2002). The effect of handling damage, mobility, body size, and fluctuating asymmetry on lifetime mating success of Ischnura graellsii (Rambur)(Zygoptera: Coenagrionidae). Odonatologica, 31 (2), 117-128.

Daniel Kissling, W., Pattemore, D. E., \& Hagen, M. (2014). Challenges and prospects in the telemetry of insects. Biological Reviews, 89 (3), 511-530. doi:doi: 10.1111/brv.12065

Denwood, M. J. (2016). runjags: An R package providing interface utilities, model templates, parallel computing methods and additional distributions for MCMC models in JAGS. Journal of Statistical Software, 71 (9), 1-25. doi:doi: 10.18637/JSS.V071.I09 
Dickinson, M. H., Lehmann, F.-O., \& Sane, S. P. (1999). Wing Rotation and the Aerodynamic Basis of Insect Flight. Science, 284 (5422), 1954-1960. doi:doi: 10.1126/science.284.5422.1954

Fujiwara, M., Anderson, K. E., Neubert, M. G., \& Caswell, H. (2006). On the estimation of dispersal kernels from individual mark-recapture data. Environmental and Ecological Statistics, 13 (2), 183. doi:doi: 10.1007/s10651-005-0007-z

Garbin, V. (2018). The Micro-World Observed by Ultra High-Speed Cameras. In: Springer.

Gimenez, O., Bonner, S. J., King, R., Parker, R. A., Brooks, S. P., Jamieson, L. E., . . . Thomas, L. (2009). WinBUGS for population ecologists: Bayesian modeling using Markov Chain Monte Carlo methods. In Modeling demographic processes in marked populations (pp. 883-915): Springer.

Gimenez, O., Rossi, V., Choquet, R., Dehais, C., Doris, B., Varella, H., . . . Pradel, R. (2007). State-space modelling of data on marked individuals. ecological modelling, 206 , 431-438.

Hagler, J. R., \& Jackson, C. G. (2001). Methods for Marking Insects: Current Techniques and Future Prospects. Annual Review of Entomology, 46 (1), 511-543. doi:doi: 10.1146/annurev.ento.46.1.511

Hammill, E., \& Clements, C. F. (2020). Imperfect detection alters the outcome of management strategies for protected areas. Ecology letters, 23 (4), 682-691. doi:doi: 10.1111/ele.13475

Hassall, C., Sherratt, T. N., Watts, P. C., \& Thompson, D. J. (2015). Live fast, die old: no evidence of reproductive senescence or costs of mating in a damselfly (Odonata: Zygoptera). Journal of Animal Ecology, 84 (6), 1542-1554. doi:doi: 10.1111/1365-2656.12407

Kery, M., \& Schaub, M. (2011).Bayesian population analysis using WinBUGS: a hierarchical perspective : Academic Press.

LaBarbera, M. (1989). Analyzing body size as a factor in ecology and evolution. Annual review of Ecology and Systematics, 20 (1), 97-117. doi:doi: 10.1146/annurev.es.20.110189.000525

Lailvaux, S. (2018). Feats of Strength: How Evolution Shapes Animal Athletic Abilities : Yale University Press.

Lebreton, J.-D., Burnham, K. P., Clobert, J., \& Anderson, D. R. (1992). Modeling survival and testing biological hypotheses using marked animals: a unified approach with case studies. Ecological monographs, 62 (1), 67-118. doi:doi: 10.2307/2937171

Lettink, M., \& Armstrong, D. P. (2003). An introduction to using mark-recapture analysis for monitoring threatened species. Department of Conservation Technical Series A, 28 , 5-32.

Li, Q., Zheng, M., Pan, T., \& Su, G. (2018). Experimental and Numerical Investigation on Dragonfly Wing and Body Motion during Voluntary Take-off. Scientific Reports, 8 (1), 1011. doi:doi: 10.1038/s41598-01819237-w

Maie, T., Wilson, M. P., Schoenfuss, H. L., \& Blob, R. W. (2009). Feeding kinematics and performance of Hawaiian stream gobies, Awaous guamensis and Lentipes concolor: linkage of functional morphology and ecology. Journal of morphology, 270 (3), 344-356. doi:doi: 10.1002/jmor.10695

Marras, S., Noda, T., Steffensen, J. F., Svendsen, M. B., Krause, J., Wilson, A. D., . . . Domenici, P. (2015). Not so fast: swimming behavior of sailfish during predator-prey interactions using high-speed video and accelerometry. Integrative and comparative biology, 55 (4), 719-727. doi:doi: 10.1093/icb/icv017

Martins, E. G., Hinch, S. G., Patterson, D. A., Hague, M. J., Cooke, S. J., Miller, K. M., . . . Farrell, A. P. (2011). Effects of river temperature and climate warming on stock-specific survival of adult migrating Fraser River sockeye salmon (Oncorhynchus nerka). Global Change Biology, 17 (1), 99-114. doi:doi: 10.1111/j.13652486.2010.02241.x 
Morton, A. (1982). The effects of marking and capture on recapture frequencies of butterflies. Oecologia, 53 (1), 105-110.

Muijres, F. T., Johansson, L. C., Bowlin, M. S., Winter, Y., \& Hedenstrom, A. (2012). Comparing aerodynamic efficiency in birds and bats suggests better flight performance in birds. PloS one, 7 (5), e37335. doi:doi: 10.1371/journal.pone.0037335

Ozgul, A., Bateman, A. W., English, S., Coulson, T., \& Clutton-Brock, T. H. (2014). Linking body mass and group dynamics in an obligate cooperative breeder. Journal of Animal Ecology, 83 (6), 1357-1366. doi:doi: 10.1111/1365-2656.12239

Paulson, D. (2009). Dragonflies and Damselflies of the West : Princeton University Press.

Plummer, M. (2003). JAGS: A program for analysis of Bayesian graphical models using Gibbs sampling.Paper presented at the Proceedings of the 3rd international workshop on distributed statistical computing.

Plummer, M. (2019). rjags: Bayesian Graphical Models using MCMC. $R$ package version 4-10., https://CRAN.R-project.org/package=rjags .

Pradel, R. (1996). Utilization of capture-mark-recapture for the study of recruitment and population growth rate. Biometrics (52), 703-709.

R Development Core Team. (2020). R: A Language and Environment for Statistical Computing. Vienna, Austria, R Foundation for Statistical Computing.

Reis, P. M., Jung, S., Aristoff, J. M., \& Stocker, R. (2010). How cats lap: water uptake by Felis catus . Science, 330 (6008), 1231-1234. doi:doi: 10.1126/science.1195421

Rico-Guevara, A., \& Mickley, J. (2017). Bring your own camera to the trap: An inexpensive, versatile, and portable triggering system tested on wild hummingbirds.Ecology and evolution, 7 (13), 4592-4598. doi:doi: 10.1002/ece3.3040

Rose, J. P., Wylie, G. D., Casazza, M. L., \& Halstead, B. J. (2018). Integrating growth and capture-markrecapture models reveals size-dependent survival in an elusive species. Ecosphere, 9 (8), e02384. doi:doi: $10.1002 / \mathrm{ecs} 2.2384$

Service, M. (1993). Mark-Recapture Techniques and Adult Dispersal. Mosquito Ecology: Field Sampling Methods , 652-751.

Sherratt, T. N., Hassall, C., Laird, R. A., Thompson, D. J., \& Cordero-Rivera, A. (2011). A comparative analysis of senescence in adult damselflies and dragonflies (Odonata).Journal of Evolutionary Biology, 24 (4), 810-822. doi:doi: 10.1111/j.1420-9101.2010.02222.x

Sherratt, T. N., Laird, R. A., Hassall, C., Lowe, C. D., Harvey, I. F., Watts, P. C., \& et al. (2010). Empirical evidence of senescence in adult damselflies (Odonata: Zygoptera). Journal Animal Ecology, 79 , 1034-1044. doi:doi: 10.1111/j.1365-2656.2010.01719.x

Steen, R. (2014). The use of a low cost high speed camera to monitor wingbeat frequency in hummingbirds (Trochilidae). Ardeola, 61 (1), 111-120. doi:doi: 10.13157/arla.61.1.2014.111

Stoks, R. (2001). Male-biased sex ratios in mature damselfly populations: real or artefact?Ecological Entomology, 26 (2), 181-187. doi:doi: 10.1046/j.1365-2311.2001.00301.x

Su, Y.-S., \& Yajima, M. (2020). R2jags: Using R to Run 'JAGS'. R package version 0.6-1, https://CRAN.Rproject.org/package $=$ R2jags .

Tufto, J., Lande, R., Ringsby, T.-H., Engen, S., Saether, B.-E., Walla, T. R., \& DeVries, P. J. (2012). Estimating Brownian motion dispersal rate, longevity and population density from spatially explicit markrecapture data on tropical butterflies. Journal of Animal Ecology, 81 (4), 756-769. doi:doi: 10.1111/j.13652656.2012.01963.x 
Zajitschek, F., Zajitschek, S., \& Bonduriansky, R. (2020). Senescence in wild insects: Key questions and challenges. Functional Ecology, 34 (1), 26-37. doi:doi: 10.1111/1365-2435.13399

\section{Figure caption}

Figure 1. Still images taken from high-speed videos showing the mark on the hindwing of the two studied dragonflies. (a) Anax junius and (b) Rhionaeshna multicolor. These marks on the wing are typically not detectable to the naked human eye when these dragonflies are in flight.

Figure 2. Resighting probabilities of females and males of the two dragonfly species monitored during capture-mark-recapture using conventional observations (CV) and integrating high-speed-videos (CV+HSV). (a) Anax junius . (b) Rhinoaeschna multicolor. Error bars are the $95 \%$ credible intervals.

Figure 3. The effect of age on the survival probability of the two dragonfly species monitored during capturemark-recapture using conventional observations (CV: blue) and integrating high-speed-videos (CV+HSV: red). (a) Anax junius . (b) Rhinoaeschna multicolor . Error bars are the $95 \%$ credible intervals

Figure 4. The effect of wing length on the survival probability of the two dragonfly species monitored during capture-mark-recapture using conventional observations (CV: blue) and integrating high-speed-videos (CV+HSV: red). (a) Anax junius . (b)Rhinoaeschna multicolor. Error bars are the $95 \%$ credible intervals

\section{Supplementary video}

Video S1. Samples of high-speed videos taken during the capture-mark-recapture study of Anax junius andRhionaeschna multicolor. The high-speed videos are slowed down 10 times.
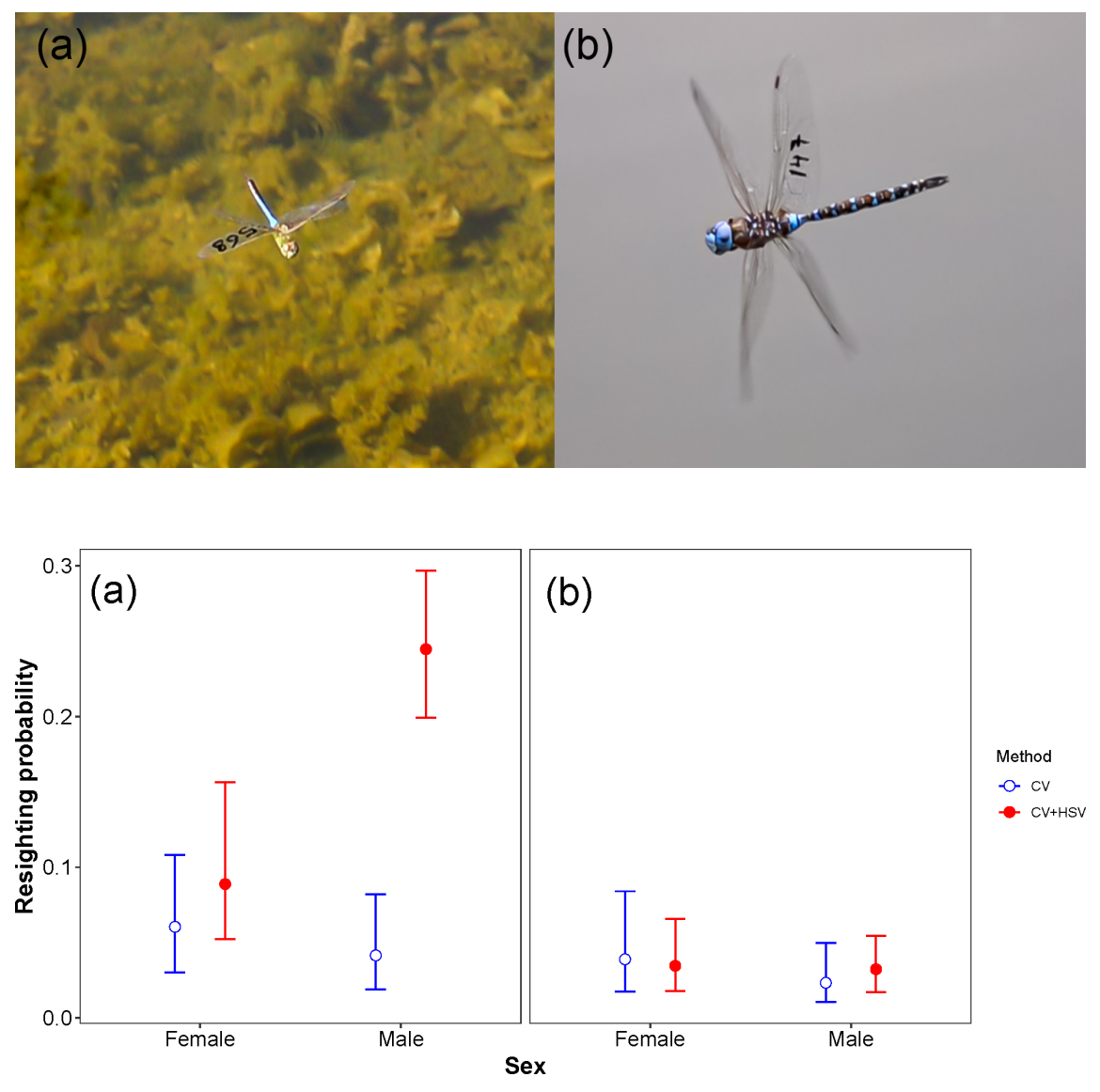

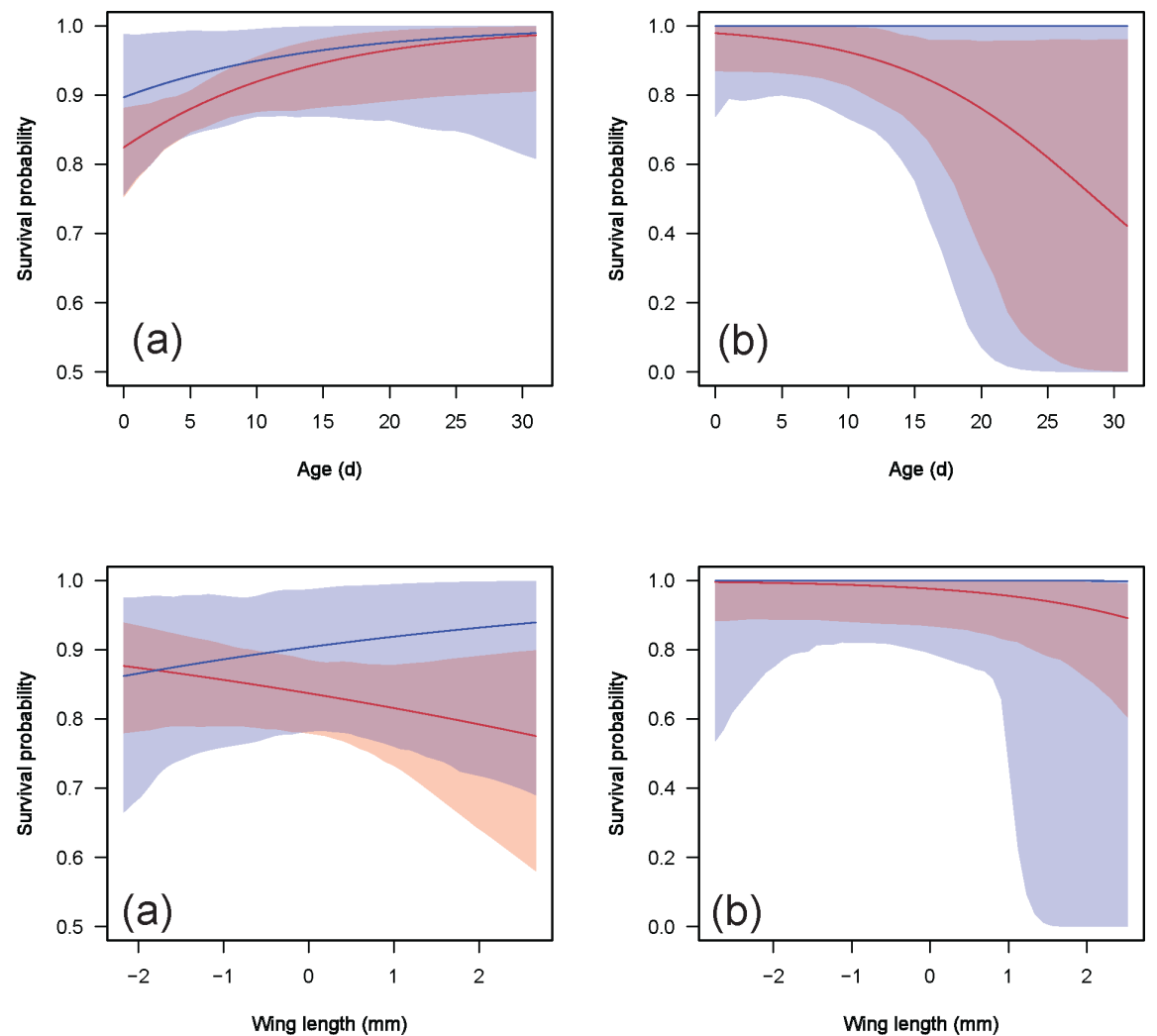